\title{
1 Over-expression of integrin $\beta 3$ can partially overcome the defect of integrin $\beta 3$ signaling in transglutaminase 2 null macrophages
}

\author{
Beáta Tóth ${ }^{\mathrm{a}}$, Zsolt Sarang ${ }^{\mathrm{a}}$, György Vereb ${ }^{\mathrm{b}}$, Ailiang Zhang ${ }^{\mathrm{c}}$, Sakae Tanaka ${ }^{\mathrm{d}}$, \\ Gerry Melino ${ }^{\mathrm{e}, \mathrm{f}}$, László Fésüs ${ }^{\mathrm{a}}$, Zsuzsa Szondy ${ }^{\mathrm{a}, *}$ \\ a Department of Biochemistry and Molecular Biology, Apoptosis and Genomics Research Group, Hungarian Academy of Sciences, \\ University of Debrecen, Nagyerdei krt. 98, Debrecen H-4012, Hungary \\ ${ }^{\mathrm{b}}$ Department of Biophysics and Cell Biology, Research Center of Molecular Medicine, University of Debrecen, Debrecen H-4012, Hungary \\ ' University of Edinburgh/Medical Research Council Centre for Inflammation Research, The Queen's Medical Research Institute, Edinburgh EH16 4TJ, UK \\ d Department of Orthopaedic Surgery, Faculty of Medicine, The University of Tokyo, Tokyo 113 0033, Japan \\ e Fondazione S. Lucia, Roma, Italy \\ ${ }^{\mathrm{f}}$ Medical Research Council, Toxicology Unit, Hodgkin bld, Leicester LE1 9HN, UK
}

\section{A R T I C L E I N F O}

Article history:

Received 12 February 2009

Received in revised form 10 July 2009

Accepted 20 July 2009

Available online $\mathrm{xxx}$

Keywords:

Macrophages

Apoptotic cells

Transglutaminase 2

Integrin signaling

\begin{abstract}
A B S T R A C T
Transglutaminase 2 (TG2) is a protein crosslinking enzyme with many additional biological functions. We have previously shown that in $\mathrm{TG} 2^{-1-}$ mice the in vivo clearance of apoptotic cells is defective leading to autoimmunity. TG2 contributes to the formation of phagocytic portals by binding to both integrin $\beta_{3}$, a known phagocytic receptor, and its bridging molecule, MFG-E8. In TG2 null macrophages integrin $\beta_{3}$ cannot accumulate around the apoptotic cells and its signaling is impaired. In the present study we describe a subline of TG2 null mice, in which a compensatory increase in integrin $\beta_{3}$ expression, which resulted alone in a high receptor concentration around the apoptotic cells without the requirement for accumulation, partially corrected the defect in integrin $\beta_{3}$ signaling. Our data provide a proof for the concept that the function of TG2 is to stabilize accumulated integrin $\beta_{3}$ concentration in the phagocytic cup.
\end{abstract}

(C) 2009 Published by Elsevier B.V.

\section{Introduction}

Apoptotic cells are generated by diverse physiological processes, ranging from the elimination of damaged (or precancerous) cells to deletion of cells during developmental morphogenesis [1]. The culmination of the apoptotic program in vivo is the phagocytosis of the apoptotic cell. In mammals prompt removal of apoptotic cells is required to prevent the release of potential self-antigens and the onset of autoimmune-like syndromes [2].

A number of receptors have been identified on macrophages that either directly or indirectly facilitate apoptotic cell recognition and uptake [3]. These receptors converge on two evolutionally conserved pathways upstream to the activation of the low molecular weight GTPase Rac1, which is obligatorily required for the uptake [4]. One of these receptors is the integrin $\alpha_{v} \beta_{3}$ (vitronectin receptor), which is bridged via the milk fat globulin EGF factor 8 (MFG-E8) molecule to the phosphatidylserine appearing on apoptotic cells [5]. The integrin $\beta_{3}$ pathway regulates Rac1 activity via the $180 \mathrm{kDa}$ protein downstream of chicken tumor virus no. 10 (CT10) regulator kinase II (Dock180) and the engulfment and migration protein

\footnotetext{
* Corresponding author. Tel.: +36 52416 432; fax: +36 52314989.

E-mail address: szondy@indi.dote.hu (Z.Szondy).
}

(ELMO), which form together an unconventional two-part guanine nucleotide exchange factor for Rac1 [6]. The formation of the DOCK180/ELMO complex is induced in the integrin $\beta_{3}$ pathway by the Trio-controlled RhoG-GTP [7]. Rac activation then leads to the formation of an actin-rich phagocytic cup, followed by the internalization of the target [8].

In addition to its role in the phagocytosis of apoptotic cells, the RhoG-mediated pathway also participates in cell migration, and is transiently activated wherever lamellopodias are formed [9] resulting in the formation of a basal Rac-GTP level in the continually migrating macrophages.

Transglutaminases are a family of thiol- and $\mathrm{Ca}^{2+}$-dependent acyl transferases that catalyze the formation of a covalent bond between the $\gamma$-carboxamide groups of peptide-bound glutamine residues and various primary amines, including the $\varepsilon$-amino group of lysine in target proteins [10]. Eight distinct enzymatically active transglutaminases have so far been described [11]. TG2 is very unique among the TG family members, because besides catalyzing the formation of protein crosslinks, it is also a $\mathrm{G}$ protein, and possesses protein disulfide isomerase and protein kinase activities [12]. In addition, TG2 also interacts with integrins of the $\beta_{1}$ and $\beta_{3}$ subfamilies, and integrin/TG2 complexes are detected inside the cell during biosynthesis and accumulate as coreceptors on the cell surface $[13,14]$. TG2 can also bind to the major extracellular protein 
fibronectin [15], and cooperate with integrins in cell adhesion and motility through either a direct noncovalent interaction with the $\beta_{1}$ and $\beta_{3}$ integrin subunits or formation of stable ternary complexes with integrins and fibronectin [13,14]. These interactions induce integrin clustering and modify integrin signaling [16].

We have previously reported that $\mathrm{TG} 2^{-1-}$ mice develop an agedependent autoimmunity due to defective in vivo clearance of apoptotic cells [17]. Recently we found that TG2 expressed on the cell surface of macrophages promotes the engulfment of apoptotic cells by forming a complex with both integrin $\beta_{3}$ and MFG-E8 [18]. In the absence of TG2 the apoptotic cell-induced integrin $\beta_{3}$ signaling leading to RhoG and Rac1 activation is impaired. Additionally, we also described that the uptake of apoptotic cells by wild-type macrophages occurs via one or two phagocyte portals that are characterized by the accumulation of phagocytic receptors in the phagocytic cup and concentration of Rac molecules to this pole of the macrophages. In the absence of TG2 both the formation and the uptake rate of these portals are less efficient than that of wild-type macrophages. TG2 null macrophages try to compensate the loss of TG2 by increasing the expression of both integrin $\beta_{3}$ and RhoG [18]. Here we describe a subline of TG2 null mice, in which a strong compensatory increase in integrin $\beta_{3}$ expression was able to correct partially the defect in integrin $\beta_{3}$ signaling.

\section{Materials and methods}

\subsection{Antibodies and reagents}

Purified mouse anti-Rac1 monoclonal (clone 102), and phycoerythrine (PE)-conjugated anti-mouse $\beta_{3}$ integrin (clone 2C9.G2) antibodies were purchased from BD Pharmingen (San Diego, CA). 6-Carboxy-3',6'-diacetylfluorescein (CFDA), 5-(and 6-)-(((4-chloromethyl)benzoyl)amino) tetramethylrhodamine (CMTMR), Alexa 647-conjugated goat anti-mouse IgG, and Prolong antifade reagent were purchased from Invitrogen (Carlsbad, CA). Horseradish peroxidase-conjugated anti-rabbit, anti-goat and antimouse IgGs, anti-mouse IgG-FITC, and vitronectin were purchased from Sigma-Aldrich (Budapest, Hungary). Anti-RhoG (clone C-20) antibodies were purchased from Santa Cruz Biotechnology (Santa Cruz, CA). The enhanced chemiluminescence for immunoblots analysis was purchased from Millipore (Millipore, Billerica, MA).

\subsection{Cell culture}

$\mathrm{TG}^{+/+}$and $\mathrm{TG}^{-/-}$[19] mice were injected with $2 \mathrm{ml} 4 \%$ thioglycollate and 4 days later macrophages were obtained by peritoneal lavage. Macrophages were allowed to adhere in 24 -well plates and non-adherent cells were washed away. For phagocytosis assays, macrophages were stained overnight with CMTMR $(10 \mu \mathrm{M})$. Thymocytes from 4 weeks old WT mice were used as apoptotic target cells. Thymi were mechanically disrupted, and isolated thymocytes were labeled overnight with CFDA $(6 \mu \mathrm{M})$. To induce apoptosis cells were treated with $4 \mu \mathrm{M}$ ionomycin (Sigma-Aldrich) for $6 \mathrm{~h}$, at which time, $40-50 \%$ of thymocytes were Annexin $V$ positive (i.e. apoptotic), and less than $5 \%$ of Annexin $\mathrm{V}$ positive cells were propidium iodide positive (i.e. necrotic). Thymocytes and macrophages were cultured in RPMI 1640 medium supplemented with $10 \%$ FCS, L-glutamine, penicillin and streptomycin (Gibco, Grand Island, NY). The study protocol was approved by Animal Care Committee of University of Debrecen.

\subsection{Phagocytosis assay}

CMTMR-stained macrophages were incubated with apoptotic thymocytes labeled with CFDA in 40:1 target/macrophage ratio for $1 \mathrm{~h}$. Cells incubated with apoptotic thymocytes incubated at $4{ }^{\circ} \mathrm{C}$ were used as controls. After washing, the cells on the plate were trypsinized, resuspended in cold medium with $0.5 \%$ sodium azide, and 10,000-20,000 cells were analyzed for each point by two-color flow cytometry (Epix Coulter) to determine the percentage of double labeled (engulfing) macrophages.

\subsection{Immunofluorescence staining and confocal microscopy}

Peritoneal macrophages isolated from WT and TG2 ${ }^{-1-}$ mice were plated in two-well chamber-slides $\left(5 \times 10^{5} /\right.$ well $)$ and cultured for $48 \mathrm{~h}$ before use. After co-culturing macrophages with apoptotic cells for $30 \mathrm{~min}$, cells were washed, fixed in ethanol/acetone $1: 1$ for $10 \mathrm{~min}$ at $-20^{\circ} \mathrm{C}$. For integrin $\beta_{3}$ staining macrophages were blocked with $50 \% \mathrm{FBS}$ for $30 \mathrm{~min}$ at $37^{\circ} \mathrm{C}$, then washed with ice cold HEPES buffer and stained with PE-conjugated anti-mouse $\beta_{3}$ integrin antibody for $15 \mathrm{~min}$ on ice. For intracellular staining, cells were permeabilized with $0.1 \%$ Triton $\mathrm{X}$ after fixation and blocked with $1 \%$ BSA. For detecting Rac1 cells were labeled with purified mouse anti-Rac1 monoclonal antibody for $30 \mathrm{~min}$ at room temperature. After washing, Alexa 647-conjugated goat anti-mouse IgG was used as a secondary antibody. Images were taken with a Zeiss LSM 510 or Olympus FV1000 confocal laser scanning microscope. For visualizing the distribution of integrin $\beta_{3}$ and Rac1, overview images and 3D stacks were acquired at $1 \mu \mathrm{m}$ optical thickness. 3D reconstructions and XYZ projections were created with the LSM 4.0 software.

\subsection{Q-PCR analysis for detecting the expression of various phagocytosis receptors}

Total RNA was isolated by TRI reagent (Sigma-Aldrich). Total RNA concentrations were determined by spectrometry after DNAse treatment (Sigma-Aldrich). TaqMan Reverse Transcription Reagent Kit (Applied Biosystems, Foster City, CA) was used for generating cDNA according to manufacturer's instructions. 200 ng total RNA was used in a reaction volume of $50 \mu \mathrm{l}$. ABI PRISM 7700 Sequence Detection System (Applied Biosystems) was used to determine the relative gene expression. Gene primers and probes were designed and supplied by Applied Biosystems. 18S ribosomal RNA was used as an endogenous control to normalize the amount of the sample cDNA added to the reaction. The $18 \mathrm{~S}$ primers were labeled with VIC and sample primer with FAM. All samples were run in triplicate. Relative mRNA expression was quantified by comparing the cycle threshold (CT) between control and knockout cell samples.

\subsection{Detection of active Rac1 and RhoG}

Macrophages plated overnight were exposed to $2 \mu \mathrm{m}$ carboxylated latex beads for $40 \mathrm{~min}$, or left untreated as controls, to detect GTP-bound forms of Rac1 and RhoG. Pull-down assay was performed with the EZ-Detect Rac1 Activation Kit (Pierce, Rockford, IL) according to manufacturer's instruction. For the RhoG pulldown assay PAK-GST was replaced with an ELMO-GST protein. Active RhoG was detected by Western blot analysis using antiRhoG antibody. For these assays $1-1.5 \mathrm{mg}$ of total cell proteins (determined by the Bradford method) was used in the pulldowns.

\subsection{Adenoviral gene delivery system}

Recombinant, replication-deficient adenoviral vectors encoding either LacZ and the murine wild-type or constitutively active Rac1 [20] gene were produced using the AdEasy XL system (Stratagene) according to the manufacturer's instruction. Virus titers were determined by plaque assay in 293 cells after exposing them to 
virus for $48 \mathrm{~h}$ in DMEM medium supplemented with $2 \%$ serum and antibiotics. For gene delivery, $10^{6}$ macrophages were exposed to the indicated amount of virus particles for $48 \mathrm{~h}$ in the same medium. LacZ expression was determined with X-gal staining, while Rac1 expression by Western blot analysis using anti-Rac1 specific antibodies.

\subsection{Time-lapse video}

For time-lapse video macrophages were or were not labeled with CMTMR as above and were exposed to unlabeled apoptotic thymocytes in a 6 target: 1 macrophage ratio. For detecting the formation of 3-phosphoinositides in the cellular membranes, cells were transfected by a fusion protein consisting of GFP and the $\mathrm{PH}$ domain of phospholipase $\mathrm{C} \delta$ by adenoviral gene delivery [21]. Time-lapse movies were made using both fluorescence emission (in the standard rhodamine channel) and transmission of green $(543 \mathrm{~nm})$ light in a Zeiss LSM510 confocal laser scanning microscope equipped with a $40 \times / 1$.2NA water immersion objective. $1024 \times 1024$ pixel images were taken every $10 \mathrm{~s}$ at $140 \mathrm{~nm} / \mathrm{pixel}$ resolution. Regions of interest were extracted and exported to mpg format with 16 frames/s speed, yielding a compressed jpg video 160 times the actual speed of the process of phagocytosis.

\subsection{Statistical analysis}

All the data are representative of at least three independent experiments. Values are expressed as mean \pm S.D. Statistical analysis was performed using the unpaired Student's $t$-test.

\section{Results}

3.1. Macrophages from a subline of TG2 null mice have very low phagocytic activity

To generate enough TG2 null macrophages for the phagocytosis experiments, we crossed TG2 null mice with each other through several generations and noticed that after a one year period of crossing macrophages from these mice had a lower phagocytic activity than macrophages from those TG2 null mice which were originated from crossing heterozygous mice. While following exposure to apoptotic cells in 40:1 target cells:macrophage ratio for $1 \mathrm{~h}$ $45 \pm 12 \%$ of TG2 null macrophages were able to engulf at least one apoptotic cell [ 18 ], only $25 \pm 8 \%(p<0.05)$ of the macrophages from this subline had phagocytic activity. Then we looked by confocal microscopy at the number of apoptotic cells engulfed by individual macrophages. While we have found that phagocyting wild-type (Fig. 1a) and TG2 null macrophages engulfed various numbers of apoptotic cells [18], macrophages from this subline engulfed predominantly one single apoptotic cell (Fig. 1b). In most of the cases the uptake even of this single apoptotic cell has not been finished following a $1 \mathrm{~h}$ phagocytosis, as it is illustrated in a 3D reconstruction (Fig. 1c).

\subsection{Macrophages from a subline of TG2 null mice have altered morphology}

Not only the phagocytic capacity, but the appearance of the macrophages from this subline has also dramatically changed. (a)

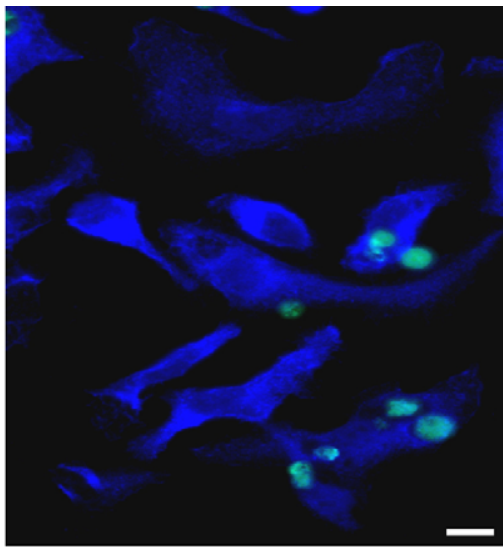

(b)

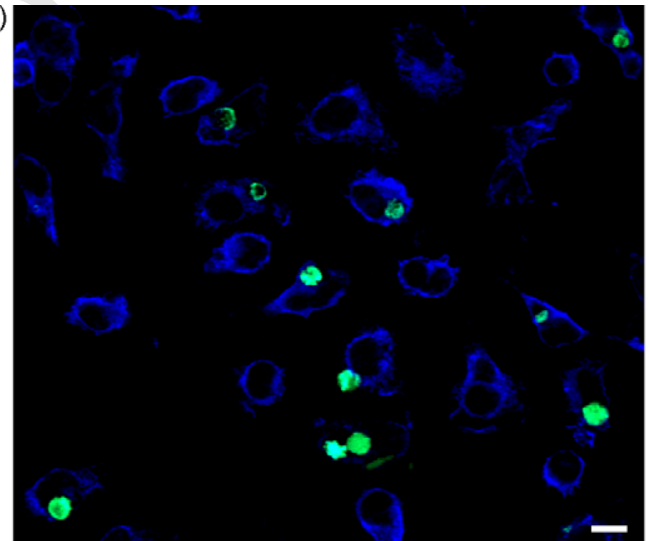

(c)

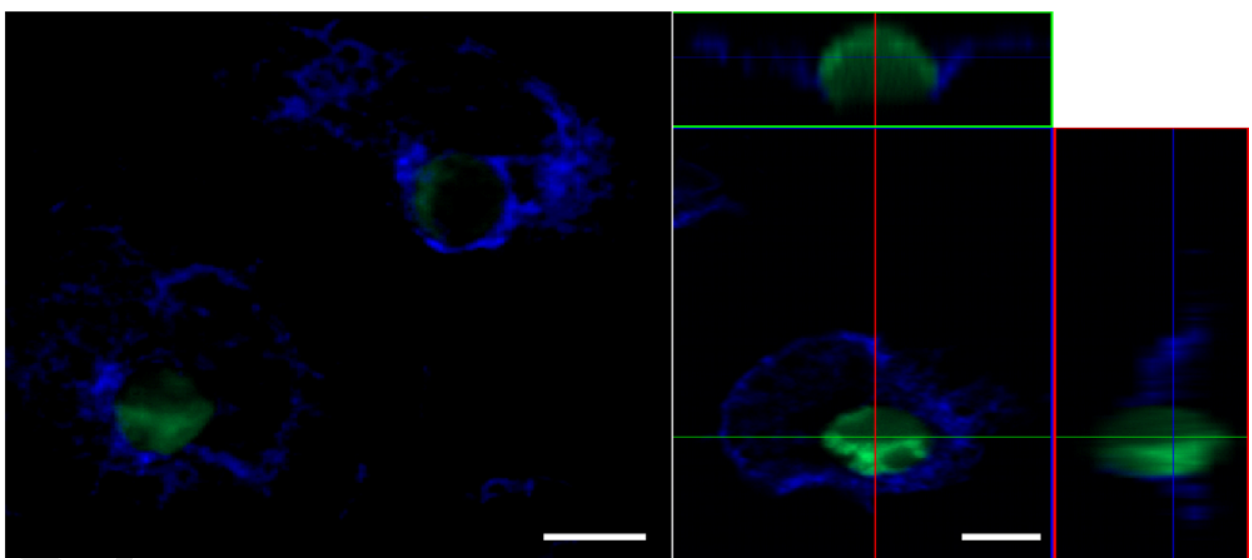

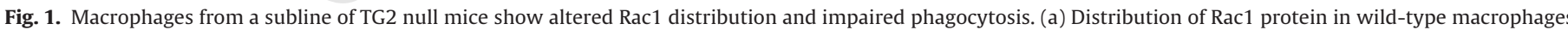

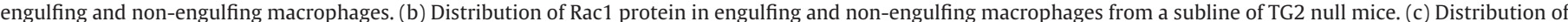

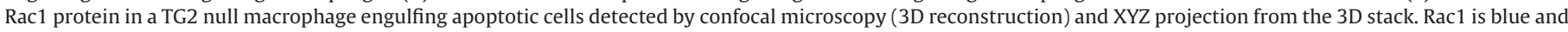

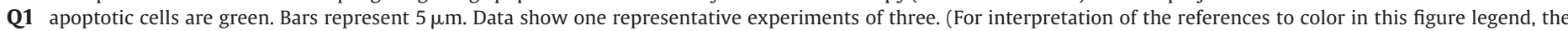
reader is referred to the web version of this article.)

Please cite this article in press as: Tóth B, et al. Over-expression of integrin $\beta 3$ can partially overcome the defect of integrin $\beta 3$ signaling in transglutaminase 2 null macrophages. Immunol Lett (2009), doi:10.1016/j.imlet.2009.07.009 


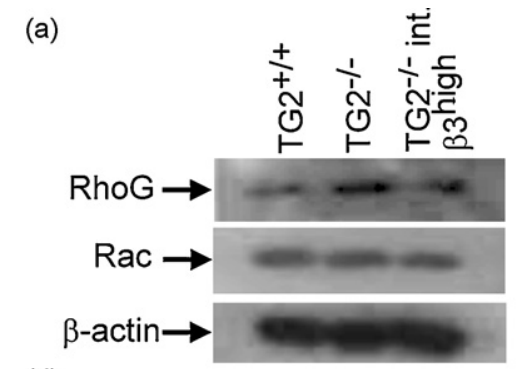

(b)

(d)

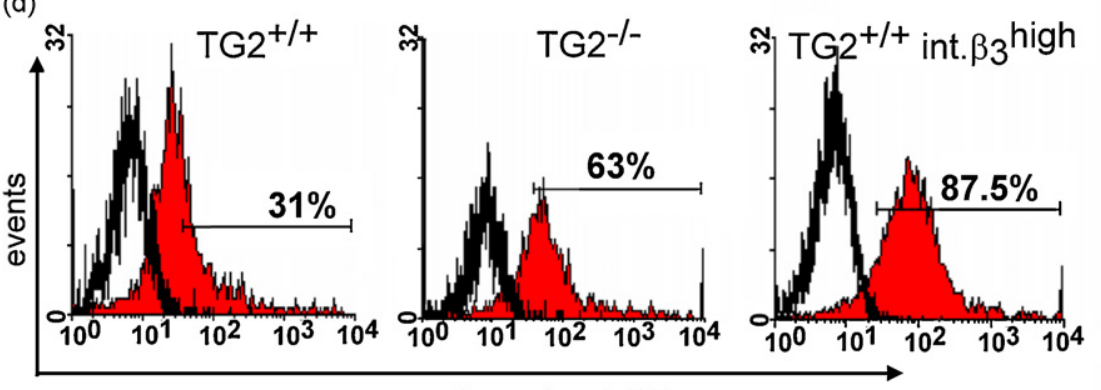

integrin $\beta 3-P E$

(e)
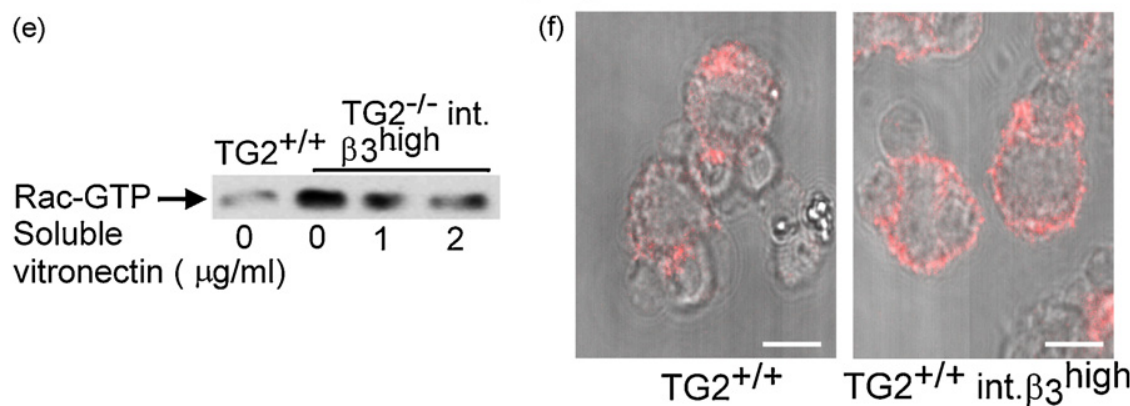

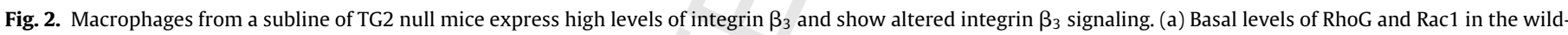

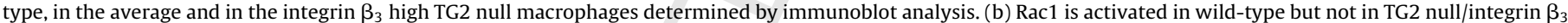

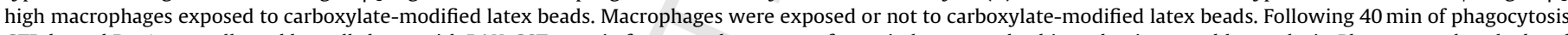

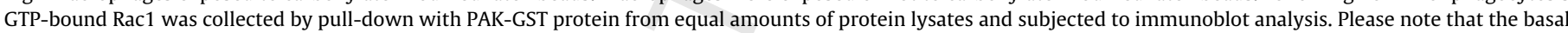

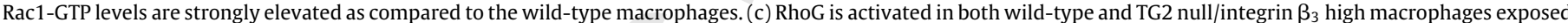

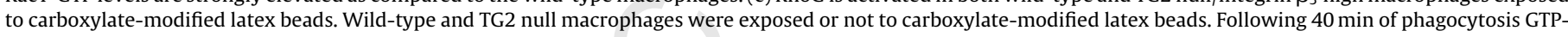

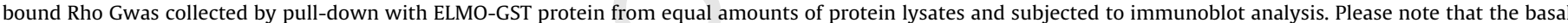

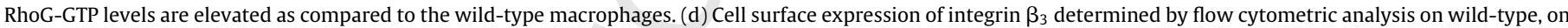

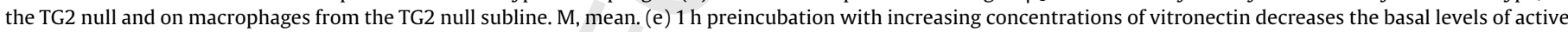

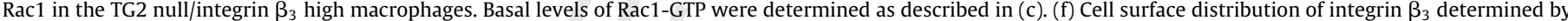

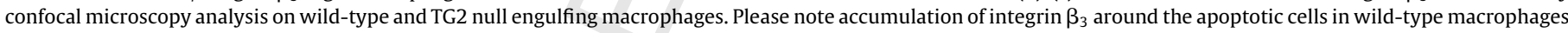
(arrows), while the even distribution of it in TG2 null/integrin $\beta_{3}$ macrophages.

While non-engulfing WT (Fig. 1a) and TG2 null cells had fibroblastoid forms [18], and Rac1 was located in the cytoplasm in the perinuclear region [18], in non-engulfing macrophages of the TG2 subline a clear recruitment of Rac1 to the periphery could be detected (Fig. 1b). This was accompanied by an enhanced lamellopodia formation and motility, which as compared to wild-types (Video 1) seemed to inhibit rather than to promote phagocytosis of apoptotic cells (Videos 2 and 3).

\subsection{Altered macrophage morphology in the TG2 null subline is related to an enhanced integrin $\beta 3$ signaling}

Since enhanced motility and membrane localization of Rac1 is related generally to Rac1 activation [9], we decided to determine the levels of active Rac1 in these macrophages with PAK pull-down assay. As shown in Fig. 2a, the absolute levels of Rac1 have not changed in these TG2 null macrophages as compared to wild-type or to the average TG2 null macrophages. However, the amount of active Rac1 was significantly elevated (Fig. 2b).

Since integrin $\beta_{3}$ signaling can be coupled to Rac1 activation leading to enhanced motility via activating RhoG [9], we decided to determine the basal and active levels of RhoG as well as the cell surface levels of integrin $\beta_{3}$. As shown in Fig. 2a, basal RhoG levels were elevated in all types of TG2 null macrophages, as compared to the wild-types. However, while RhoG-GTP levels were not elevated in the TG2 null macrophages [18], in the macrophages from the TG2 null subline an elevated RhoG-GTP level was detected (Fig. 2c).

Concomitantly, much higher levels of integrin $\beta_{3}$ were found on the cell surface of these macrophages (Fig. 2d). When these macrophages were kept in the presence of increasing concentrations of soluble vitronectin, which competes with the binding of the immobilized integrin $\beta_{3}$ ligands and thus interferes with the signaling [22,23], the levels of active Rac1 were decreased proving that the elevated amount of Rac1-GTP levels are indeed a consequence of the enhanced integrin $\beta_{3}$ levels and signaling in these cells (Fig. 2e).

\subsection{Apoptotic cells can induce the activation of RhoG in macrophages from the TG2 subline}

Previous studies have shown that apoptotic cells cannot activate RhoG and Rac1 of the integrin $\beta_{3}$ signaling pathway in TG2 null 
(a)

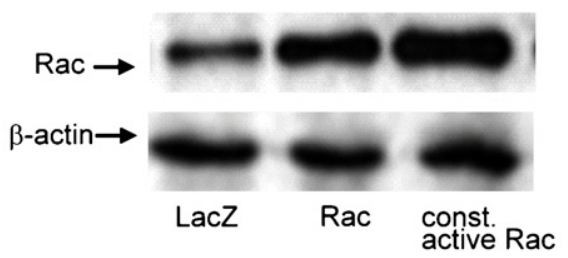

(c)

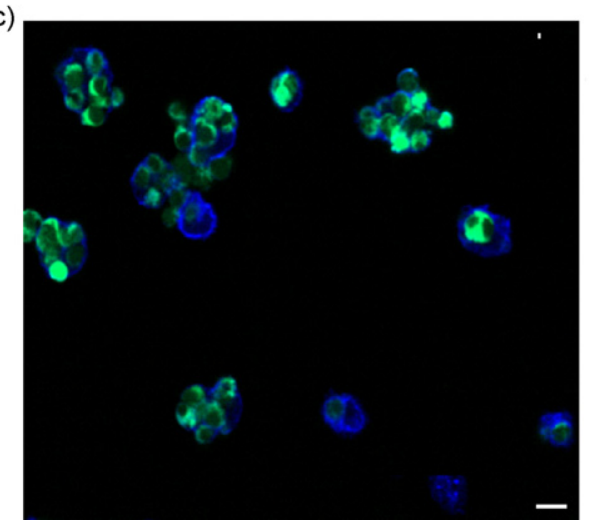

(b)

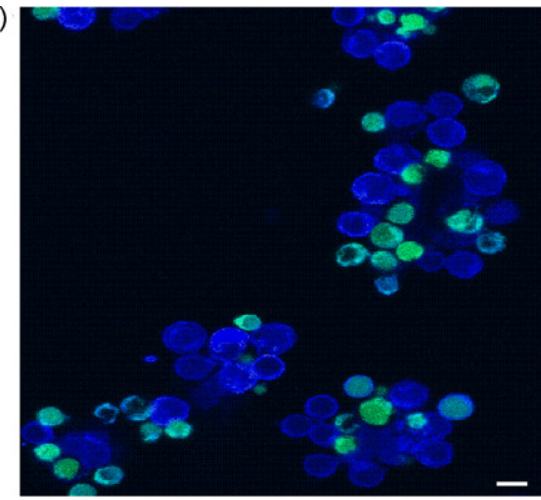

(d)

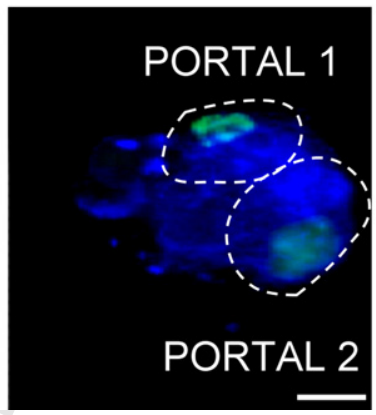

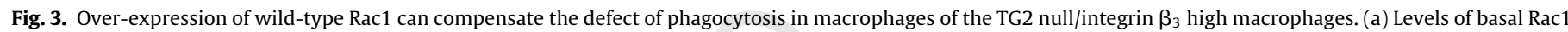

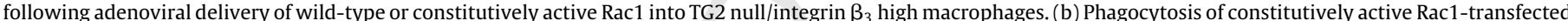

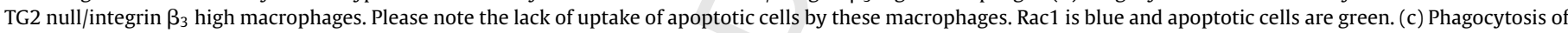

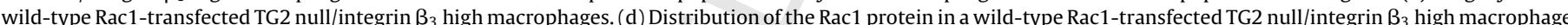

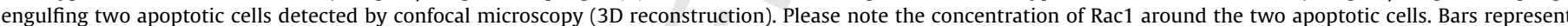
$5 \mu \mathrm{m}$. (For interpretation of the references to color in this figure legend, the reader is referred to the web version of this article.)

macrophages [18]. But in the macrophages of this subline increased expression of integrin $\beta_{3}$ could maintain an elevated Rac1 level. That is why we decided to test the functionality of the integrin $\beta_{3}$ pathway during phagocytosis of apoptotic cells. In context with our previous observations [18] macrophages of this TG2 subline also did not concentrate integrin $\beta_{3}$ around the apoptotic cells (Fig. 2f). However, due to the increased receptor levels, intense stainings of integrin $\beta_{3}$ could be detected all around the macrophage including the phagocytic cup. Next the activation of RhoG and Rac1 was tested $[4,6,7]$. Since GTP-bound Rac1 in apoptotic cells could interfere with the pull-down assay, we used 2 - $\mu$ m carboxylate-modified latex beads in these studies, which have been previously used as surrogate apoptotic cells in signaling studies [24]. A shown in Fig. 2c, when macrophages from the TG2 subline were exposed to carboxylate-modified latex beads the amount of active RhoG increased indicating that increased levels of integrin $\beta_{3}$ compensated the loss of TG2 in the phagocytosis signaling at least at the level of RhoG activation. However, the elevated Rac1-GTP levels could not be further increased when macrophages were exposed to carboxylate-modified latex beads (Fig. 2b). In context with these observations, when these macrophages were exposed to apoptotic cells, Rac1 remained recruited all over the periphery and did not accumulate around the apoptotic cells (Fig. 1b and c).

\subsection{Over-expression of wild-type Rac1 can compensate the defect of phagocytosis in macrophages of the TG2 null subline}

There are two possible explanations for the lack of Rac1 activation by apoptotic cells. Either TG2 is required for proper apoptotic cell-induced signaling to activate Rac1, which is not compen- sated by the enhanced levels of integrin $\beta 3$, or as a result of the TG2-independent, but integrin $\beta 3$-dependent enhanced motility the amount of free Rac1, which could be activated and localized around the apoptotic cells, is exhausted. To test whether providing of additional Rac1 molecules could overcome the defect in integrin $\beta_{3}$ signaling, macrophages from the TG2 null subline were transfected with wild-type or constitutively active Rac1 molecules by adenoviral gene delivery, and their phagocytosis was detected by FACS analysis. Addition of constitutively active Rac1 (Fig. 3a) completely inhibited the phagocytosis of apoptotic cells (Fig. 3b), indicating that switching on and off of Rac1 is required for proper phagocytosis of apoptotic cells. However, when these cells were transfected with wild-type Rac1 (Fig. 3a), Rac1 accumulation could be detected around the apoptotic cells to form the efficient phagocytic portal (Fig. 3d). Consequently the phagocytosis rate of the Rac1-transfected cells reached that of the wild-type cells ( $75 \pm 12 \%$ and $82 \pm 7 \%$ for wild-type and TG 2 null/integrin $\beta_{3}$ high macrophages, respectively). Not only the percentage of the engulfing macrophages has increased following Rac1 transfection, but Rac1-transfected TG2 null macrophages also efficiently took up apoptotic cells, though their enhanced motility has remained (Fig. 3c, Video 4). In contrast, transfection of wild-type Rac1 into average TG2 null macrophages resulted only in a mild increase of the phagocytosis rate (from $45 \pm 12 \%$ to $56 \pm 13 \%$ ).

Our previous studies have demonstrated that wild-type macrophages take up apoptotic cells via one or two portals. As demonstrated in Fig. 3c and Video 5, Rac1-transfected TG2 null/integrin $\beta_{3}$ high macrophages engulf apoptotic cells also via two portals, indicating that not the number of integrin $\beta 3$ molecules determine the number of phagocytic portals. 


\subsection{Apoptotic cell-induced formation of 3-phosphoinositides is} impaired in TG2 null macrophages

Activation of phosphatidylinositol-3-OH kinase (PI-3kinase) is required for proper phagocytosis [25], and both DOCK180 and ELMO contain recognition domains for 3-phosphoinositides for proper membrane localization and thus directing Rac1 activation [26]. To detect the formation of 3-phosphoinositides during the phagocytosis of apoptotic cells both wild-type (Video 6) and TG2 null macrophages (Video 7) were transfected by PLC $\delta$-PHD-GFP. Apoptotic cell recognition induced formation of 3-phosphoinositides around the apoptotic cells in wild-type macrophages, while this induction was impaired in TG2 null macrophages indicating that not only the activation of RhoG, but proper activation of the phosphatidylinositol-3-OH kinase is also under the control of TG2.

\section{Discussion}

Previous studies done in our laboratory have shown that the in vivo loss of TG2 results in impaired phagocytosis of apoptotic cells leading to autoimmunity [17]. The loss of TG2 affected the macrophage side, where TG2 seems to act as an integrin $\beta_{3}$ coreceptor interacting with both integrin $\beta_{3}$ and its phagocytosis ligand MFG-E8 [18]. While in wild-type macrophages integrin $\beta_{3}$, similarly to other phagocytic receptors, is concentrated in the phagocytic cup, in the absence of TG2, integrin $\beta_{3}$ was evenly distributed on the macrophage cell surface and the apoptotic cell-induced signaling of integrin $\beta_{3}$ leading to RhoG and Rac1 activation was impaired. TG2 null cells try to compensate the loss of TG2 by upregulating the expression of both integrin $\beta_{3}$ and its signaling partner RhoG to enhance the efficiency of integrin $\beta_{3}$ signaling in the absence of TG2.

Wild-type macrophages use one or two phagocyte portals to engulf apoptotic cells. Loss of TG2 did not affect the number of phagocytic portals, but in the absence of TG2 both the formation and the engulfment rate of the phagocyte portals formed were less efficient. So we proposed that the role of TG2 is to stabilize the phagocyte portals [18].

In the present study we describe a subline of TG2 null mice, in which the macrophages compensated the loss of TG2 by a much higher expression of integrin $\beta_{3}$ than the average TG2 null macrophages. Integrin $\beta_{3}$ and RhoG signaling is involved not only in the phagocytosis of apoptotic cells, but also in cell motility [9]. As a result, increased expression of integrin $\beta_{3}$ in these cells resulted in enhanced motility with high basal active RhoG and Rac1 levels. Concomitant with the elevated basal active Rac1 levels, in nonengulfing TG2 null/integrin $\beta_{3}$ high macrophages Rac1 was located mostly bound to the cellular membranes, while we found it in the cytosol in wild-type and in regular TG2 null macrophages [18]. When these macrophages were exposed to apoptotic cells, integrin $\beta_{3}$ remained evenly distributed on the cell surface, but, due to the enhanced expression a high density was detected around the apoptotic cells as well. In these cells addition of carboxylate-modified latex beads, unlike in regular TG2 null macrophages [18], triggered the activation of RhoG, indicating that the elevated integrin $\beta_{3}$ levels were sufficient to overcome the defect caused by the loss of TG2 in the initiation phase of integrin $\beta_{3}$ signaling. Still activation of Rac1, a downstream target of RhoG, could not be observed, and the activation of PI-3kinase was impaired. Concomitantly, we could not detect a significant accumulation of Rac1 around the apoptotic cells, and the phagocytosis of apoptotic cells was more severely affected.

Though integrin $\beta_{3}$ and RhoG are involved in both cell motility and phagocytosis, TG2 is not equally required for both. While phagocytosis of apoptotic cells is enhanced [18], integrin- dependent migration on laminin, for example, is inhibited [27] by TG2. Thus the loss of TG2 might not equally affect the phagocytic and motility signaling pathways, especially that of the activation of PI-3kinase, which seems to be independent of integrin signaling in the context of phagocytosis (Raymund Birge, personal communication). Moderate increase in integrin $\beta_{3}$ expression observed in TG2 null macrophages is expected to enhance the impaired phagocytosis in the absence of TG2 and to induce enhanced motility, as it enhances the number of integrin receptors and the efficiency of its signaling. However, if the levels of integrin $\beta_{3}$ increase further, the two pathways might become competitive, integrin-induced motility winning by being too efficient in Rac1 activation and using up free Rac1. This is because total RhoG levels adapted to the changes in integrin $\beta_{3}$ expression and enhanced integrin signaling, but there were no alterations in the basal Rac1 levels. This was suggested by the findings that while basal GTP-bound RhoG levels of the TG2 null/integrin $\beta_{3}$ high macrophages were lower than the ones induced by surrogate apoptotic cells in wild-type macrophages and remained inducible (Fig. 2c), basal levels of the GTP-bound Rac1 much exceeded the ones that were detectable in wild-type cells exposed to the surrogate apoptotic cells (Fig. 2b). So we decided to test by transfection of Rac1 molecules, whether the efficiency of the phagocytosis signaling could be altered by increasing the levels of the free Rac1, which forms the next step in the integrin $\beta_{3}$ signaling pathway. Transfection of constitutively active Rac1 completely inhibited the phagocytosis of apoptotic cells. This observation confirms that of Nagaya et al. [28] and suggests that dynamic switching on and off of Rac1 is required for proper phagocytosis. Addition of wild-type Rac1, however, efficiently increased the rate of phagocytosis. These macrophages were able to concentrate additional Rac1 around the apoptotic cells and engulfed apoptotic cells with a similar rate as wild-type macrophages.

Our data provide a further proof for the hypothesis that the role of TG2 in phagocytosis is to provide efficient integrin $\beta_{3}$ signaling around the apoptotic cells (either by promoting integrin $\beta_{3}$ clustering in the phagocytic cup or by enhancing the affinity of the receptor for its ligand MFG-E8/phosphatidylserine), which in the absence of TG2 can also be achieved by an enhanced receptor density. Our data, however, might also suggest that TG2 is required for additional signaling pathways involved in the activation of PI-3kinase. Based on our data, integrin signaling will not affect the number of phagocytic poles formed, but as it was suggested by others, it might be critical in the initiation of the formation of the cellular pole [29].

\section{Acknowledgements}

This work was supported by Hungarian grants from the National Research Fund (OTKA T T049445, K 77587 and TS-44798) and Ministry of Welfare T (115/2006). Zsolt Sarang received a Bólyai fellowship. The ELMO-GST protein was provided by Raymond Birge. The PLC $\delta$-PH-GFP plasmid was a generous gift of T. Balla. We thank János Roszik for his help at some LSM measurements. The excellent technical assistance of Edit Komóczi and Zsolt Hartman is gratefully acknowledged.

\section{Appendix A. Supplementary data}

Supplementary data associated with this article can be found, in the online version, at doi:10.1016/j.imlet.2009.07.009.

\section{References}

[1] Henson PM, Hume DA. Apoptotic cell removal in development and tissue homeostasis. Trends Immunol 2006;27:244-50.

[2] Michlewska S, McColl A, Rossi AG, Megson IL, Dransfield I. Clearance of dying cells and autoimmunity. Autoimmunity 2007;40:267-73. 
G Model

IMLET 48241-7

B. Tóth et al. / Immunology Letters $x x x$ (2009) $x x x-x x x$

7

453

[3] Cavil J, Dransfield I, Gregory C, Haslett C. A blast from the past: clearance of apoptotic cells regulates immune responses. Nat Rev Immunol 2002;2:965-75.

[4] Kinchen JM, Cabello J, Kringle D, Wong K, Freichtinger R, Schnabel H, et al. Two pathways converge at CED-10 to mediate actin rearrangement and corpse removal in C. elegant. Nature 2005;43:93-9.

[5] Hanayama R, Tanaka M, Miwa K, Shinohara A, Iwamatsu A, Nagata S. Identificatimon of a factor that links apoptotic cells to phagocytes. Nature 2002;417:182-7.

[6] Brugnera E, Haney L, Grimsley C, Lu M, Walk SF, Tosello-Tampont AC, et al. Unconventional Rac-GEF activity is mediated through the Dock180-ELMO complex. Nat Cell Biol 2002;4:74-582.

[7] deBakker CD, Haney LB, Kinchen JM, Grimsley C, Lu M, Klingele D, et al. Phagocytosis of apoptotic cells is regulated by a UNC-73/TRIO-MIG-2/RhoG signaling module and armadillo repeats of CED-12/ELMO. Cir Biol 2004;14:2208-16.

[8] Bustelo XR, Sauzeau V, Berenjeno IM. GTP-binding proteins of the Rho/Rac family: regulation, effectors and functions in vive. Bioessays 2007;29:356-70.

[9] Katoh H, Hiramoto K, Negishi M. Activation of Rack by Rho regulates cell migration. J Cell Sci 2006;119(Pt 1):56-65.

[10] Greenberg CD, Birckbichler PJ, Rice RH. Transglutaminases: multifunctional cross-linking enzymes that stabilize tissues. FASEB J 1991;5:3071-7.

[11] Lorand L, Graham RM. Transglutaminases: crosslinking enzymes with pleiotropic functions. Nat Rev Mol Cell Biol 2003;4:140-56.

[12] Fésüs L, Piacentini M. Transglutaminase 2: an enigmatic enzyme with diverse functions. Trends Biochem Sci 2002;27:534-9.

[13] Akimov SS, Krylov D, Fleischman LF, Belkin A. Tissue transglutaminase is an integrin-binding adhesion coreceptor for fibronectin. J Cell Biol 2000;148:825-38.

[14] Akimov SS, Belkin AM. Cell surface tissue transglutaminase is involved in achesion and migration of monocytic cells on fibronectin. Blood 2001;98:1567-76.

[15] Hang J, Zemskov EA, Loran L, Belkin AM. Identification of a novel recognition sequence for fibronectin within the $\mathrm{NH2}$-terminal beta-sandwich domain of tissue transglutaminase. J Biol Chem 2005;280:23675-83.

[16] Janiak A, Zemskov EA, Belkin AM. Cell surface transglutaminase promotes Rho activation via integrin clustering and suppression of the Src-p190RhoGAP signailing pathway. Mol Biol Cell 2006;17:1606-19.

[17] Szondy Z, Sarong Z, Molnár P, Németh T, Piacentini M, Mastroberardino PG, et al. TGase $2^{-1-}$ mice reveal a phagocytosis-associated crosstalk between macrophages and apoptotic cells. Proc Natl Aced Sci USA 2003;100: 7812-7.

[18] Tóth B, Garabuczi E, Sarong Z, Vereb G, Vámosi G, Aeschlimann D, et al. Transglutaminase 2 is needed for the formation of an efficient phagecyte portal in macrophages engulfing apoptotic cells. J Immunol 2009;182: 2084-92.

[19] De Laurenzi V, Melino G. Gene disruption of tissue transglutaminase. Mol Cell Biol 2001;21:148-55

[20] Fukuda A, Hikita A, Wakemaya H, Akiyama T, Oda H, Nakamura K, et al. Regulation of osteoclast apoptosis and motility by small GTPase binding protein Rack. J Bone Miner Res 2005;20:2245-53.

[21] Varnai P, Lin X, Lee SB, Tuymetova G, Bondeva T, Spat A, et al. Inositol lipid binding and membrane localization of isolated pleckstrin homology (PH) domains. Studies on the PH domains of phospholipase C delta 1 and p130. J Biol Chem 2002;277:27412-22.

[22] Preissner KT. Structure and biological role of vitronectin. Ann Rev Cell Biol $1991 ; 7: 275-310$.

[23] Cavil J, Dransfield I, Hogg N, Gregory C. Vitonectin-receptor mediated phagecytosis of cells undergoing apoptosis. Nature 1990;343:170-3.

[24] Park D, Tosello-Trampont AC, Elliott MR, Lu M, Haney LB, Ma Z, et al. BAI1 is an engulfment receptor for apoptotic cells upstream of the ELMO/Dock180/Rac module. Nature 2007;450:430-4.

[25] Leverrier Y, Okkenhaug K, Sawyer C, Bilancio A, Vanhaesebroeck B, Ridley AJ. J Biol Chem 2003;278:38437-42.

[26] Ravichandran KS, Lorenz U. Engulfment of apoptotic cells: signals for a good meal. Nat Rev Immunol 2007;7:964-74.

[27] Lentini A, Provenzano B, Caraglia M, Shevchenko A, Abbruzzese A, Beninati S. Impairment of the metastatic activity of melanoma cells by transglutaminasecatalyzed incorporation of polyamines into laminin and Matrigel. Amino Acids 2008;34:251-6.

[28] Nagaya M, Kitani M, Matsuda M, Nagata S. Spatiotemporal activation of Rac1 for engulfment of apoptotic cells. Proc Natl Acad Sci USA 2008;105: 9198-203.

[29] Ho E, Irvine T, Vilk GJ, Lajoie G, Ravichandran KS, D’Souza SJ, et al. Integrinlinked kinase interactions with ELMO2 modulate cell polarity. Mol Biol Cell 2009;20:3033-43.

488
48
490
49
49
493
494
495
496
497
498
499
500
50
50
50
50
50
50
50
50
50
510
511
512
513
51
51
51
51
51
51
520
52
52
523

Please cite this article in press as: Tóth B, et al. Over-expression of integrin $\beta 3$ can partially overcome the defect of integrin $\beta 3$ signaling in transglutaminase 2 null macrophages. Immunol Lett (2009), doi:10.1016/j.imlet.2009.07.009 